\title{
THE PROPERTIES OF BLAZARS DETECTED BY EGRET
}

\author{
J.R. MATTOX \\ Boston University Astronomy Department \\ 725 Commonwealth Avenue, Boston, MA 02215 \\ http://bu-ast.bu.edu/ mattox/
}

\section{The Detection of Blazars by EGRET}

One of the most exciting recent developments in astrophysics is the detection of members of the blazar class of AGN at $\sim 1 \mathrm{GeV}$ by the EGRET instrument aboard the Compton Observatory. Although a weak detection of 3C 273 was obtained by the COS $B$ mission, the very strong detection of 3C 279 by EGRET (Hartman et al. 1992) two months after launch was not anticipated.

Another $\sim 50$ blazars have subsequently been identified with EGRET sources. Detected blazars include BL Lacertae objects, "Highly Polarized Quasars" (HPQs), and "Optically Violent Variable" quasars (OVVs). Analysis of the EGRET data for the initial 3C 279 observation revealed strong variability on $\sim 1$ day timescales (Kniffen et al. 1993). Variability is also apparent for all of the other bright EGRET blazars (Mattox et al. 1997a). The brightest EGRET blazar, PKS 1622-297, shows variability on a time scale of several hours (Mattox et al. 1997b), as rapid as possibly resolved with the meager EGRET statistics. The EGRET blazar detections led to Čerenkov observations which have detected $\mathrm{TeV} \boldsymbol{\gamma}$-rays from nearby $\mathrm{BL}$ Lac objects Mrk 421 (Gaidos et al. 1996) and Mrk 501 (Catanese et al. 1997) which have both been observed to flare dramatically. Von Montigny et al. (1995) and Mukherjee et al. (1997) provide compendia of EGRET blazar results. Chiang et al. (1996) report evidence for positive evolution, and present a luminosity function.

A careful analysis of EGRET source identifications is appropriate because the location of $\gamma$-ray emission can't be accurately determined with EGRET. In the first EGRET catalog (Fichtel et al. 1994), the reliability of the blazar identification was not addressed. In the second EGRET cata$\log$ (Thompson et al. 1995 \& 1996), blazar identifications were classified as 
"high-confidence" if the radio source was located within the $95 \%$ confidence contour of the EGRET likelihood position estimate (Mattox et al.1997a). A similar classification is made by Punsley (1997). Thompson et al. (1995 $\& 1996)$ classified some radio sources beyond the $95 \%$ confidence contour as "lower-confidence" identifications. Mattox et al. (1997a) offer a thorough analysis of the identification of EGRET sources with radio sources. They find that there is no doubt that EGRET is detecting blazars - they specify 16 EGRET blazar identifications for which the confidence of a correct identification exceeds of $99 \%$. They also tabulate an additional $\sim 50$ plausible identifications. The third EGRET catalog (Hartman et al. 1997) will change the status of some plausible EGRET blazar identifications because additional EGRET exposure has been used to refine position estimates of previously detected sources, and new EGRET sources have been detected.

\section{The Emission of Gamma Rays by Blazars}

The apparent $\gamma$-ray luminosity of the EGRET blazars (as quantified by $\nu F_{\nu}$ ) is often 1-2 orders of magnitude greater than that observed at other energies. Therefore, unless the $\gamma$-ray emission is more highly beamed than that at lower frequencies, most of the non-thermal energy emerges as $E \gtrsim 100$ $\mathrm{MeV}$ photons during the $\gamma$-ray high states. The rapid variability of the flux observed by EGRET implies that the emission takes place in a compact region. Because the opacity to $\gamma-\gamma$ pair production must be low for the $\gamma$-rays to escape, relativistic beaming is required if the observed $\mathrm{x}$-rays originate in the same volume as the $\gamma$-rays (Dondi \& Ghisellini 1995). Blazar 3C 279 was observed in 1996 to flare simultaneously at $\gamma$-ray and x-ray energies (Wehrle et al. 1998). This indicates that $\mathrm{x}$-rays do originate in approximately the same volume as $\gamma$-rays, and that the $\gamma$-rays are produced in a relativistic jet.

Most models of blazar $\gamma$-ray emission feature inverse Compton scattering as the $\gamma$-ray emission mechanism, but there is not a consensus as to the origin of the low energy photons which are scattered. It has been suggested that they might originate within the jet as synchrotron emission (Maraschi Ghisellini \& Celotti 1992). This is designated as the synchrotron self-Compton (SSC) process. Another possibility is that the scattered lowenergy photons are ambient. This is designated as the external Compton scattering (ECS) process. Dermer, Schlickeiser, \& Mastichiadis (1992) suggested that they come directly from an accretion disk around a blackhole at the base of the jet. It was subsequently proposed that the dominant source of the low energy photons for scattering could be due to re-processing of disk emission by broad emission line clouds (Sikora, Begelman, \& Rees 1994; Blandford \& Levinson 1995). Ghisellini \& Madau (1996) suggest that 
the dominant source of scattered low-energy photons is broad-line-cloud re-processing of jet synchrotron emission.

Maraschi et al. (1994) reported that the change in the multiwavelength spectrum of 3C 279 between the 1991 flare and a low state observed 18 months later was consistent with the SSC model for $\gamma$-ray emission. However, Hartman et al. (1996) noted that the spectra of 3C 279 in both these high and low states could also be fit with an ECS model. The situation is now further complicated by the finding that the spectral change for the 1996 flare of 3C 279 is different than for the 1991 flare (Wehrle et al. 1998). At the peak of the 1996 flare, the EGRET flux is a factor of 3 larger than at the peak of the 1991 flare, but the optical flux is only $70 \%$ of that at the peak of the 1991 flare. Wehrle et al. (1998) note that the $\gamma$-ray emission increases by more than the square of the observed increase of the infraredoptical flux during the course of the flare. This is probably not consistent with a one-zone SSC model. It is also not consistent with any of the ECS models except that of Ghisellini \& Madau (1996). There model can be tested with sensitive multi-epoch observations of the Ly $\alpha$ flux of 3C 279 in conjunction with simultaneous observations of the synchrotron flux of the jet (Wehrle et al. 1998).

\section{The Properties of EGRET Blazars}

Even though EGRET has obtained some exposure for all directions on the sky, only $\sim 10 \%$ of blazars are detected. Insight into the $\gamma$-ray emission process of blazars may potentially be gained by an examination of the properties that distinguish the EGRET blazars from the general blazar population. All EGRET blazars which have been observed with VLBI sufficiently well show superluminal motion (Barthel et al. 1995). Several groups are making additional VLBI observations of EGRET blazars (Tingay et al. 1996; Bower et al. 1997; Marchenko et al. 1997).

Impey (1996) reported that the total radio flux of the EGRET blazars tends to be larger than the radio flux of other blazars in the parent population. This difference is also apparent for the milliarcsecond-scale radio emission of EGRET blazars (Moellenbrock et al. 1996, Mattox et al. 1997). This supports arguments that the $\gamma$-ray emission is taking place at the base of a relativistic jet. Although it is expected to be of insufficient strength to cause the observed difference, the increase in the security of EGRET blazar identifications with radio flux (as quantified by Mattox et al. 1997a) is a selection effect which causes some bias in the observed direction. 


\section{Future $\gamma$-ray Observations}

The $\gamma$-ray emission of blazars is not yet fully understood. Because its sparkchamber gas is nearly depleted, EGRET is unlikely to provide definitive new blazar data. Data from the proposed GLAST satellite, which offers a sensitivity $1-2$ orders of magnitude better than EGRET, will be very important for further blazar studies, e.g., the GLAST data will provide for a determination of whether the diffuse extragalactic high-energy $\gamma$-ray flux (Sreekumar et al. 1997) is exclusively emitted by blazars. In the mean time, much can probably be learned from Čerenkov $\gamma$-ray blazar observations in conjunction with simultaneous observations at lower energies.

\section{References}

Barthel, P. D., et al., 1995, ApJL, 444, L21

Blandford, R.D., \& Levinson, A., 1995, ApJ, 441, 79

Bower, G.C., et al., 1997, ApJ, 484, 118

Catanese, M., et al., 1997, ApJ, 487, L143

Chiang, J., et al., 1996, ApJ, 452, 156; errata 465, 1011

Dermer, C. D., Schlickeiser, R., \& Mastichiadis, A., 1992, A\&A 256, L27

Dondi, L., Ghisellini, G, 1995, MNRAS, 273, 583

Fichtel, C.E., et al., 1994, ApJ S, 94, 551

Gaidos, J.A., et al., 1996, Nature, 383, 319

Ghisellini, G., \& Madau, P., 1996, MNRAS, 280, 67

Hartman, R.C., et al., 1992, ApJ, 385, L1

Hartman, R.C., et al., 1996, ApJ, 461, 698

Hartman, R.C., et al., 1997, in preparation

Impey, C., 1996, AJ, 112, 2667

Kniffen, D.A., et al. 1993, ApJ, 411, 133

Maraschi, L., Ghisellini, G., \& Celotti, A. 1992, ApJ 397, L5

Maraschi, L., et al., 1994, ApJ 435, L91

Marchenko, S.G., Marscher, A.P., et al., 1997, IAU Coll. 164, ASP, in press

Mattox, J.R., et al., 1997a, ApJ, 481, 95

Mattox, J.R., et al., 1997b, ApJ, 476, 692

Moellenbrock, G.A., 1996, AJ, 111, 2174

von Montigny, C., et al., 1995, ApJ, 440, 525

Mukherjee R., et al., 1997, ApJ, 490, 116

Punsley, B., 1997, AJ, 114, 544

Sikora, M., Begelman, M. C., \& Rees, M. J. 1994, ApJ 421, 153

Sreekumar, P., et al. 1997, ApJ, in press

Thompson, D. J., et al., 1995, ApJ S, 101, 259

Thompson, D. J., et al., 1996, ApJ S, 107, 227

Tingay, S.J., et al., 1996, ApJ, 464, 170

Wehrle et al., 1998, ApJ, in press 